\title{
Sign-Talk: Hand Gesture Recognition System
}

\author{
Aishwarya Auti \\ Affiliated to Savitribai \\ Phule Pune University \\ AISSMS COE, Pune,India
}

\author{
Romalee Amolic \\ Affiliated to Savitribai \\ Phule Pune University \\ AISSMS COE, Pune,India
}

\author{
Shubham Bharne \\ Affiliated to Savitribai \\ Phule Pune University \\ AISSMS COE, Pune,India
}

\author{
Ankita Raina \\ Affiliated to Savitribai \\ Phule Pune University \\ AISSMS COE, Pune,India
}

\author{
D. P. Gaikwad \\ Affiliated to Savitribai \\ Phule Pune University \\ HOD, Professor, \\ Computer Engineering \\ AISSMS COE, Pune,India
}

\begin{abstract}
The speech and hearing impaired face a severe problem of communication with the normal people as the sign language used by them, is not understood by a majority of the people. Today those using sign language require a human sign language interpreter to surpass the communication barriers with non-disabled people. Hence we will develop a system that will advance their social integration as they are enabled to express themselves to non-sign language speakers. Hence, in order to bridge the gap between the speech and hearing impaired and normal people, we are developing a system which will enable them to easily communicate with the non disabled using hand gestures and sign language. The proposal is to design an integrated hardware and software solution which will consist of leap motion controller and computer based application. We propose an efficient and real time model which recognizes the hand gestures of the impaired using leap motion controller as the primary input device. The data from the leap motion controller will be processed and transmitted to the computer application for gesture discrimination and speech translation. The input signal will be acquired and examined to see if it is a legal sign language gesture or not.
\end{abstract}

\section{Keywords}

Sign Language, Leap Motion Controller, Feature Extraction , Sign Recognition, Text-to-Speech.

\section{INTRODUCTION}

Human Computer Interaction (HCI) plays an important role in today's world as the number of computer applications are increasing rapidly. Human Computer Interaction aims to make human-machine interaction more natural, similar to the human-human interaction[1]. For hearing and speech impaired people, sign language is the primary means of communication. Hand gestures are able to represent ideas and actions, using different hand shapes (and their corresponding associated movements if any), which can be identified by the gesture recognition system[1].These are then interpreted to recognize the sign language and convert the meaning of signs into speech. Common image processing systems have problems such as unstable brightness, noise, poor resolution and contrast. In this paper Leap Motion Controller(LMC) is used for static hand gesture recognition of American Sign Language (ASL). This paper is mainly organized in following sections: Section 2 gives an introduction to the Leap Motion Controller. Section 3 is literature review of the various approaches experimented for use with hand gesture recognition systems, for sign language interpretation. Section 4 is the detailed introduction of our approach for recognition of hand gestures in order to interpret sign language using LMC. It is further followed by conclusion and future perspective.

\section{INTRODUCTION TO LEAP MOTION CONTROLLER}

The Leap Motion controller is a small USB peripheral device which includes two IR cameras and three infrared LED's.

It uses Infrared (IR) rays to define the position of predefined objects in a limited space in real time. The device range is a rough hemispherical area, to a distance of about 2 meter. Leap Motion sensor is a small sized sensor which is easy to use and inexpensive.

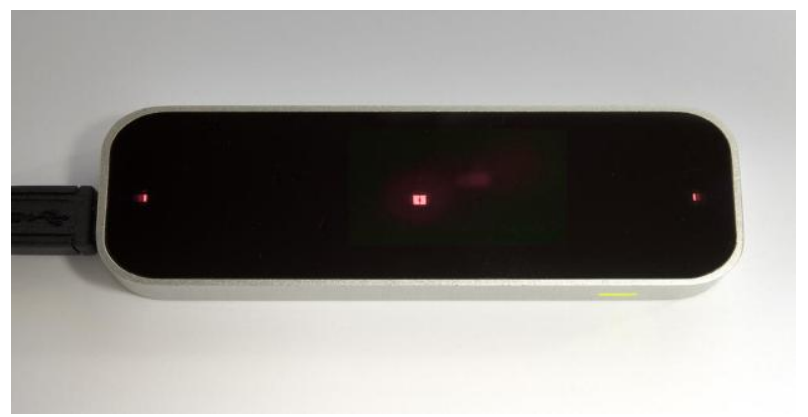

Figure 1. Leap Motion Controller

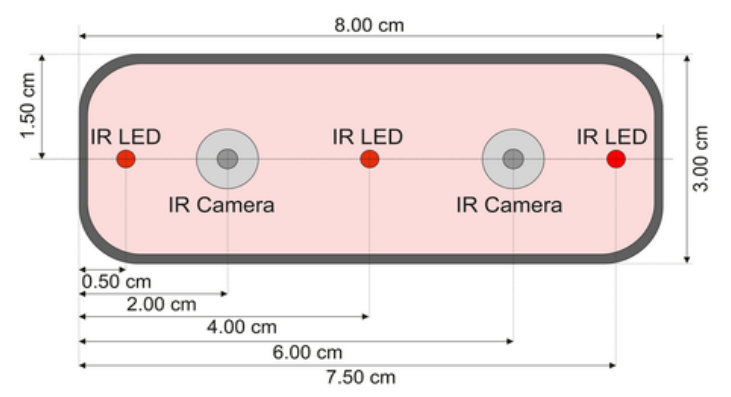

Figure 2. Internal representation of LMC

\section{LITERATURE REVIEW}

Various approaches have been used by different researchers for the recognition of different hand gestures. Hand gesture recognition is not limited to paper or digital surfaces, but has 
also been extended to the third dimension These approaches can be broadly categorized as data glove based approach, vision based approach and color based approach[2].

It has been proposed that a set of computer vision algorithms are based on the color or intensity of images. Applications like the camera mouse, which tracks a certain key point in a stream of images to control a computer's pointing device, were based on the RGB data[2]. Colored gloves are used to obtain hand shape recognition applied to the conversion of a sign language. Colored gloves dyed with 6 different colors are worn and the feature vectors are calculated using the Morphological Principal Component Analysis from captured images[4]. Hand detection along with finger modeling are performed in natural background with the help of colored gloves. The proposed feature vector extraction method makes the camera distance highly independent. To analyze the performance of feature extraction, neural network is trained for the position recognition of a sign language with 26 alphabet letters.Since it must be taken with colored gloves the glove worn is not suitable[3].

Secondly, in order to recognize static sign language, a robust approach involved using a novel combination of features.

According to the studies, in the case of colored glove based recognition, the user may feel inconvenient to wear them and a photograph must be taken whilst wearing the colored glove, which is not very troublesome. In case of Kinect based recognition of gestures, a large region is required to obtain depth and color image is necessary for the skeletal tracking. In case of Leap Motion based recognition, the machine learning requires large computation for a new person.

A simplification made in this project is the elimination of the hardware required and the use of leap motion controller which in turn increases the overall accuracy of the project considerably.

\section{METHODOLOGY}

In this system the hand gestures are tracked with the help of a leap motion controller. The leap motion controller gives various hand points as output.For each and every gesture there are many features, interesting points on the object that can be extracted to provide a "feature" description of the object[5].The features are extracted.It gives a mapping of the gesture in terms of feature points. Leap motion controller gives the tips of the fingers along with the centre of the palm as the points. The features which are extracted from the Leap motion controller are then stored using serialization. We are using serialization as the method of storing our data. Each input gesture would be stored in the form of an object. The feature vector consists of the distances between the tip of the fingers and the centre of the palm. This is the first feature vector. The second feature vector consists of the distances between the adjacent finger tips. While testing, again the gestures are captured and the positions of the finger points are extracted using methods including Euclidean distance method, Cosine, Jaccard, Dice distance measure algorithms.Initially all the gestures are first stored into the serializable database. For recognition, the feature vectors are compared using the four similarity measures namely Euclidean distance measure, Cosine similarity, Jaccard similarity and Dice similarity. The one with maximum similarity(least distance) is returned as the detected gesture[1]. Figure 3 gives a detailed view of the architecture of the system.

\subsection{Data Procurement}

The leap motion controller has a field view in which it tracks the hands and finger positions and creates a track of frames.The frame data represents a set of tracking data for hands and fingers detected in a single frame. The acquired data through the leap sensor consists of a vector of objects to store the various physical characteristics of the detected fingers such as the distances between the fingertips.

\subsection{Feature Extraction}

Figure 4 highlights the features extracted for recognition.

The extracted key points are coordinates of finger positions from the input gesture.The points are, the center of the palm(say P), tips of thumb(say T), index finger(say I), middle finger(say $M$ ), ring finger(say $R$ ), pinky finger(say $K$ ) for each hand.

The coordinates are, $\mathrm{P}(\mathrm{x} 1, \mathrm{y} 1, \mathrm{z} 1), \mathrm{T}(\mathrm{x} 2, \mathrm{y} 2, \mathrm{z} 2), \mathrm{I}(\mathrm{x} 3, \mathrm{y} 3, \mathrm{z} 3)$, $\mathrm{M}(\mathrm{x} 4, \mathrm{y} 4, \mathrm{z} 4), \mathrm{R}(\mathrm{x} 5, \mathrm{y} 5, \mathrm{z5}), \mathrm{K}(\mathrm{x} 6, \mathrm{y} 6, \mathrm{z6})$ for each hand.The extracted points are stored in a serializable database and distances between each tip and palm center is calculated with respect to each finger as shown in figure 4.

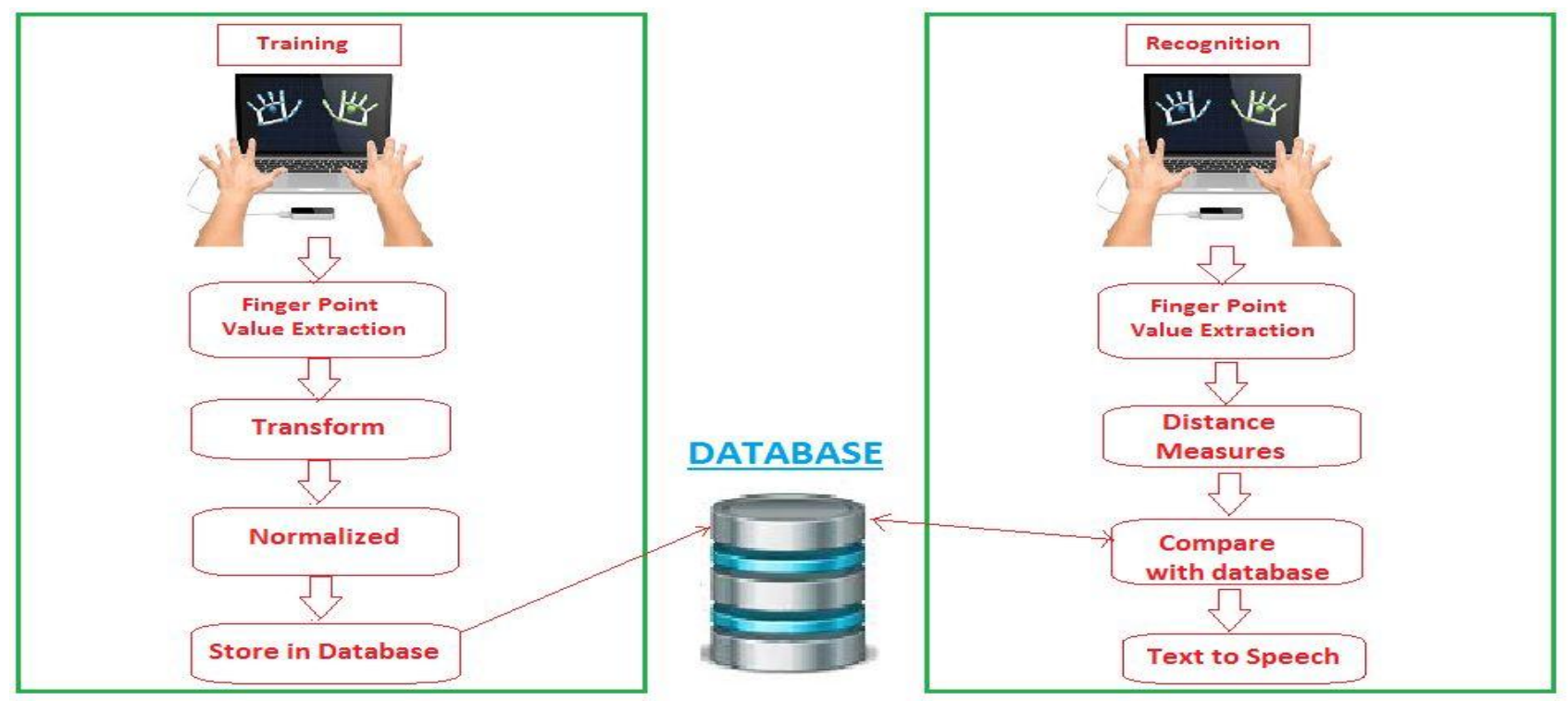

Figure 3. System Architecture 


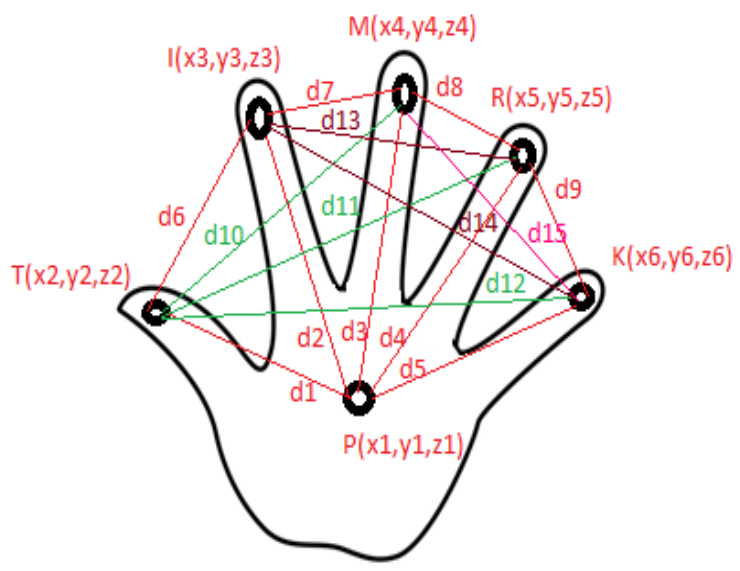

Figure 4. Feature Point Extraction

\subsection{Gesture Classification}

Whenever a gesture is performed the points are extracted and they are stored using serialization. Then the features are extracted.At the run time, distances are calculated from the extracted feature points using distance formula which gives the most efficient result amidst the four distance measure algorithms given above.The cosine distance measure algorithm gives the fastest and the most efficient measure as follows:

Cosine distance

$\mathrm{d}(\mathrm{A}, \mathrm{B})=\cos (\Theta)=(\mathrm{A} . \mathrm{B}) /\|\mathrm{A}\|\|\mathrm{B}\|$

$\mathrm{d} 1$ gives the distance between points $\mathrm{P}$ and $\mathrm{T}, \mathrm{d} 2$ gives the distance between $\mathrm{P}$ and $\mathrm{L}, \mathrm{d} 3$ gives the distance between points $\mathrm{P}$ and $\mathrm{M}, \mathrm{d} 4$ gives the distance between $\mathrm{P}$ and $\mathrm{R}, \mathrm{d} 5$ gives the distance between points $\mathrm{P}$ and $\mathrm{K}, \mathrm{d} 6$ gives the distance between points $\mathrm{T}$ and $\mathrm{L}, \mathrm{d} 7$ gives the distance between points $\mathrm{L}$ and $\mathrm{M}$, $\mathrm{d} 8$

gives the distance between points $\mathrm{M}$ and $\mathrm{R}$, $\mathrm{d} 9$ gives the distance between points $\mathrm{R}$ and $\mathrm{K}$.The detected gesture is given as the output and results are compared.

\section{Euclidean distance}

$\mathrm{d}(\mathrm{p}, \mathrm{q})=\sqrt{\left((\mathrm{q} 1-\mathrm{p} 1)^{\wedge} 2+(\mathrm{q} 2-\mathrm{p} 2)^{\wedge} 2+\cdots(\mathrm{qn}-\mathrm{pn})^{\wedge} 2\right)}$

Cosine distance

$\mathrm{d}(\mathrm{A}, \mathrm{B})=\cos (\Theta)=\frac{(\mathrm{A} * \mathrm{~B})}{|\mathrm{A}| *|B|}$

\section{Jaccard distance}

$$
d(x, y)=\frac{(|x \cap y|)}{|x|+|y|-|x \cap y|}
$$

\section{Dice distance}

$d(X, Y)=\frac{2|X \cap Y|}{|X|+|Y|}$

\subsection{Speech output}

For every gesture which is acquired the name of the gesture will be produced as the speech output.This enables the listener to hear the word as signed by the user along with the text depicting the word.

\section{EXPERIMENTAL RESULTS}

The system is tested for gestures of ASL, 26 alphabets (A to Z), 10 digits ( 1 to 10$)$ given in the following tables. Table 1 and 2 give the recognition accuracy for alphabets and numbers respectively with four similarity measures. Algorithm is tested on 10 different persons to calculate average accuracy.

Table 1: Recognition accuracy for Alphabets

\begin{tabular}{|l|l|l|l|l|}
\hline $\begin{array}{l}\text { Method/ } \\
\text { Gesture }\end{array}$ & cosine & Euclidean & Dice & Jaccard \\
\hline A & $90 \%$ & $80 \%$ & $70 \%$ & $70 \%$ \\
\hline B & $90 \%$ & $70 \%$ & $70 \%$ & $70 \%$ \\
\hline C & $100 \%$ & $100 \%$ & $100 \%$ & $100 \%$ \\
\hline D & $90 \%$ & $70 \%$ & $70 \%$ & $70 \%$ \\
\hline E & $80 \%$ & $70 \%$ & $70 \%$ & $70 \%$ \\
\hline F & $80 \%$ & $70 \%$ & $70 \%$ & $70 \%$ \\
\hline G & $80 \%$ & $70 \%$ & $70 \%$ & $70 \%$ \\
\hline H & $100 \%$ & $90 \%$ & $80 \%$ & $80 \%$ \\
\hline I & $100 \%$ & $100 \%$ & $100 \%$ & $100 \%$ \\
\hline J & $90 \%$ & $80 \%$ & $80 \%$ & $80 \%$ \\
\hline K & $80 \%$ & $80 \%$ & $80 \%$ & $80 \%$ \\
\hline L & $100 \%$ & $100 \%$ & $100 \%$ & $100 \%$ \\
\hline M & $90 \%$ & $90 \%$ & $80 \%$ & $70 \%$ \\
\hline N & $90 \%$ & $90 \%$ & $80 \%$ & $70 \%$ \\
\hline O & $100 \%$ & $90 \%$ & $90 \%$ & $90 \%$ \\
\hline P & $80 \%$ & $80 \%$ & $80 \%$ & $80 \%$ \\
\hline Q & $80 \%$ & $80 \%$ & $80 \%$ & $80 \%$ \\
\hline S & $100 \%$ & $100 \%$ & $100 \%$ & $100 \%$ \\
\hline S & $100 \%$ & $100 \%$ & $100 \%$ \\
\hline S & $100 \%$ & $100 \%$ & $100 \%$ \\
\hline & $100 \%$ & $70 \%$ & $80 \%$ \\
\hline & $100 \%$ \\
\hline
\end{tabular}




\begin{tabular}{|l|l|l|l|l|}
\hline $\mathrm{Y}$ & $100 \%$ & $90 \%$ & $80 \%$ & $80 \%$ \\
\hline $\mathrm{Z}$ & $80 \%$ & $80 \%$ & $80 \%$ & $80 \%$ \\
\hline Average & $90.76 \%$ & $86.15 \%$ & $82.69 \%$ & $81.92 \%$ \\
\hline
\end{tabular}

Table 2: Recognition accuracy for numbers

\begin{tabular}{|l|l|l|l|l|}
\hline $\begin{array}{l}\text { Method/ } \\
\text { Gesture }\end{array}$ & Cosine & Euclidean & Dice & Jaccard \\
\hline 1 & $100 \%$ & $95 \%$ & $95 \%$ & $95 \%$ \\
\hline 2 & $100 \%$ & $95 \%$ & $95 \%$ & $95 \%$ \\
\hline 3 & $100 \%$ & $95 \%$ & $95 \%$ & $95 \%$ \\
\hline 4 & $100 \%$ & $95 \%$ & $95 \%$ & $95 \%$ \\
\hline 5 & $100 \%$ & $95 \%$ & $95 \%$ & $95 \%$ \\
\hline 6 & $100 \%$ & $95 \%$ & $95 \%$ & $95 \%$ \\
\hline 7 & $100 \%$ & $95 \%$ & $95 \%$ & $95 \%$ \\
\hline 8 & $100 \%$ & $95 \%$ & $95 \%$ & $95 \%$ \\
\hline 9 & $100 \%$ & $95 \%$ & $95 \%$ & $95 \%$ \\
\hline 10 & $100 \%$ & $95 \%$ & $95 \%$ & $95 \%$ \\
\hline Average & $100 \%$ & $95 \%$ & $95 \%$ & $95 \%$ \\
\hline
\end{tabular}

\section{RESULT ANALYSIS}

\section{E Alphabets 톨 Numeric}

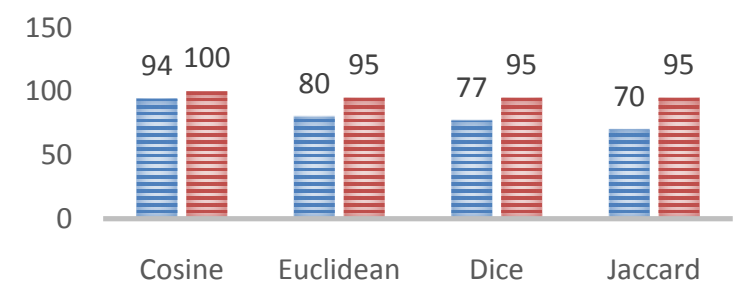

\section{CONCLUSION}

The paper presents a method to recognize static sign language gestures using a Leap motion controller. Leap motion device helps in efficient recognition of 3-D gestures.The sensor tackles the major issues in earlier systems. The database can be expanded to include the complete vocabulary of the language which may specifically include vernacular languages.In addition to that, the system can also be expanded to include dynamic gestures along with static gestures.This would provide additional flexibility and freedom to the sign language user and would facilitate the use of signs, involving patterns, to be recognised by the system.

\section{ACKNOWLEDGMENTS}

We owe the success of this paper to our guide Prof. D.P. Gaikwad for providing us with his support and guidance. He has played an instrumental role in the research and development of this topic.Most importantly, we greatly value his presence and availability.

\section{REFERENCES}

[1] Chetna Naidu, Archana Ghotkar," Hand Gesture Recognition Using Leap Motion Controller",International Journal of Science and Research (IJSR) ISSN (Online): 2319-7064

[2] Noor Adnan Ibraheem, Rafiqul Zaman Khan, " Survey on Various Gesture Recognition Technologies and Techniques",International Journal of Computer Applications (0975 - 8887) Volume 50 - No.7, July 2012

[3] Bassem Khelil, Hamid Amiri, "Hand Gesture Recognition Using Leap Motion Controller for Recognition of Arabic Sign Language". 3rd International Conference on Automation, Control, Engineering and computer Science (ACECS'16),

[4]Makiko Funasaka, Yu Ishikawa, Masami Takata, and Kazuki Joe, "Sign Language Recognition using Leap Motion Controller".Int'l Conf. Par. and Dist. Proc. Tech. and Appl. | PDPTA'15 |.

[5] Arti Thorat, Varsha Satpute, Arati Nehe, Tejashri Atre Yogesh R Ngargoje, "Indian Sign Language Recognition System for Deaf People". International Journal of Advanced Research in Computer and Communication Engineering Vol. 3, Issue 3, March 2014

[6] Viraj Shinde, Tushar Bacchav, Jitendra Pawar, Mangesh Sanap,"Hand Gesture Recognition Using Web Camera". International Journal of Advanced Engineering \& Innovative Technology, Volume 1, Issue 1, April-2014

[7] Monuri Hemantha, M.V.Srikanth, "Simulation of Real Time Hand Gesture recognition for Physically Impaired". International Journal of Advanced Research in Computer and Communication Engineering Vol. 2, Issue 11, November 2013 